\title{
La résistance, l'éducation et la culture
}

Jean-François Muracciole

\section{(2) OpenEdition}

Journals

Édition électronique

URL : http://journals.openedition.org/trema/1868

DOI : $10.4000 /$ trema. 1868

ISSN : 2107-0997

\section{Éditeur}

Faculté d'Éducation de l'université de Montpellier

\section{Édition imprimée}

Date de publication : 1 décembre 1997

Pagination : 85-98

ISSN : 1167-315X

\section{Référence électronique}

Jean-François Muracciole, "La résistance, l'éducation et la culture », Tréma [En ligne], 12-13 | 1997, mis en ligne le 01 décembre 1997, consulté le 21 avril 2019. URL : http://journals.openedition.org/ trema/1868 ; DOI : 10.4000/trema.1868

Ce document a été généré automatiquement le 21 avril 2019

Trema 


\title{
La résistance, l'éducation et la culture ${ }^{1}$
}

\author{
Jean-François Muracciole
}

1 Il peut paraître singulier d'aborder l'histoire de la résistance sous l'angle de la culture et de l'éducation. Pourtant, les résistants ont abondamment disserté sur ces matières et la moindre surprise qui attend le chercheur est de découvrir non seulement l'existence, mais l'abondance des sources. Malgré les contraintes de la clandestinité, les intellectuels résistants ont pris la peine de noircir des centaines de pages sur la diffusion de la culture, la réforme de l'enseignement ou la nécessité de fonder une nouvelle politique de la jeunesse. Ainsi, à l'été 1943, une polémique surgit sur la place du latin dans l'enseignement. Marc Bloch, l'un des principaux dirigeants de Franc-Tireuret qui devait tomber quelques mois plus tard dans les filets de la Gestapo, trouve alors le temps de rédiger sur ce thème un important article.

\section{Position de la pensée de la résistance}

2 Du côté de la résistance extérieure, la surprise est, en fait, limitée. Dans la mesure où la France libre s'est conçue très tôt comme le gouvernement légitime de la France, il était normal qu'elle développât des structures de réflexion. A Londres, dès l'été 1940, de Gaulle confia la responsabilité des "questions intellectuelles et juridiques» au juriste René Cassin et, en décembre 1941, il institua des «commissions d'étude des problèmes de l'après-guerre ». Sous l'autorité de Cassin, fut ainsi formée une « commission d'étude des problèmes intellectuels et de l'enseignement ». Présidée par Joseph Cathala, professeur de chimie à l'Université de Toulouse avant la guerre, cette commission travailla de juillet 1942 à juillet 1943. Sa composition révélait une grande diversité, reflet de l'hétérogénéité du microcosme londonien: des militaires, comme le médecin-général Sicé, des universitaires, comme Joseph Cathala ou l'historien Paul Vaucher, des personnalités liées au monde politique comme le journaliste démocrate-chrétien Maurice Schumann, le socialiste Henri Hauck ou le syndicaliste Yvon Morandat. A Alger, après la formation du CFLN, fut créée, sous l'autorité de René Capitant, commissaire du CFLN à l'Education nationale, une « commission de réforme de l'enseignement ». Cette nouvelle commission, 
présidée par l'historien Marcel Durry et composée essentiellement d'enseignants et de chercheurs, tels Francis Perrin, Louis Gernet ou Henry Laugier, travailla de mars à août 1944.

Confrontée à des conditions matérielles d'existence toutes différentes, la résistance intérieure ne put disposer de telles institutions. Elle s'efforça néanmoins de se doter de ses propres moyens de réflexion. La presse clandestine fut le lieu naturel et privilégié de la présentation des débats intellectuels de la résistance. Dès 1942, la plupart des grands mouvements de résistance disposait, à côté de leur périodique principal, de revues clandestines, le plus souvent d'une haute tenue intellectuelle, et spécialisées dans les études et la réflexion: ainsi Les Cahiers pour l'OCM, Les Cahiers de la Libération pour Libération-Sud, L'Université libre pour le Front national ou la Revue libre pour FrancTireur. A partir de l'été 1942, la formation d'organes communs à l'ensemble de la résistance facilita la diffusion de ces débats. La création, en juillet 1942, sous l'impulsion de Jean Moulin, du Comité Général d'Etudes (CGE) joua un rôle décisif en ce sens. Le CGE, qui disposait de sa propre revue, Les Cahiers politiques, avait pour mission de collecter les propositions de la résistance intérieure pour l'après-guerre et d'en proposer à Londres des synthèses.

4 Replacée dans l'évolution des grands débats culturels et éducatifs de la première moitié du siècle, la pensée de la résistance se singularise par l'originalité de son propos. On sait que les deux moments qui encadrent la période de l'Occupation ont été dominés par le thème de la popularisation de la culture. C'est de l'effervescence du Front populaire qu'a émergé, en France, le concept moderne de "politique culturelle». De même, les initiatives de la Libération, de la création d'une direction de l'Education populaire confiée à Jean Guéhenno à la fondation du Théâtre National Populaire, ont contribué à faire considérer la diffusion de la culture comme "un service public au même titre que le gaz ou l'électricité", pour reprendre la fameuse expression de Jean Vilar. Les intellectuels résistants, pour leur part, se démarquent de cette problématique. Certes, la popularisation de la culture n'est pas absente de leurs réflexions, mais le thème apparaît tardivement (pas avant l'été 1944) et au sein d'une résistance alpine dont plus d'un cadre a d'abord subi l'influence intellectuelle et politique de l'école d'Uriage. De fait, la majorité des intellectuels résistants s'est démarquée des réflexions de la période du Front populaire et, par une inflexion significative, a appréhendé les problèmes culturels sous un jour nouveau.

5 Pour les Français libres, le rayonnement culturel de la France était conçu comme un élément du combat patriotique. Affirmer la grandeur de la pensée et de l'art français, c'était démontrer que la vraie France existait encore et qu'en dépit de Vichy, elle continuait à se battre aux côtés des démocraties. La culture tendait ainsi à devenir l'instrument d'une lutte politique dont les fondements la dépassaient largement. Plus généralement, la plupart des intellectuels résistants ont opéré un élargissement du concept de « culture » qui, à leurs yeux, devait nécessairement comporter une dimension éducative. Ainsi, il est frappant de constater qu'une bonne part de leurs débats a porté sur la réforme du système éducatif. Ce glissement s'explique avant tout par le traumatisme de la défaite qui a constitué l'un des principaux points d'ancrage de la pensée résistante. Obsédés par la recherche d'une explication à cet effondrement inouï, les résistants ont fini par mettre en avant l'inadaptation du système éducatif de la III ${ }^{\mathrm{e}}$ République. 
6 Les expériences du régime de Vichy ont également influencé la formation de cette pensée. Sur la question du rôle de l'Etat, les résistants se sont retrouvés prisonniers d'un redoutable dilemme. D'un côté, la plupart d'entre eux affirmaient la légitimité et la nécessité d'un renforcement de l'initiative publique. Certains n'hésitaient d'ailleurs pas, pour ce faire, à se référer explicitement à l'œuvre fondatrice de Jean Zay et de Léo Lagrange. Mais, d'un autre côté, Vichy, par ses tentations autoritaires, sinon, aux yeux des résistants, totalitaires, avait rendu suspect le principe même de l'intervention étatique dans les domaines de la culture ou de l'éducation. Les résistants, et c'est le moindre mérite d'un René Capitant, s'employèrent alors à concevoir un cadre administratif et juridique original qui respectât à la fois le principe de l'action publique et l'indépendance des acteurs privés.

\section{De la défaite à la réforme de l'enseignement}

7 Le traumatisme de la défaite éclaire la signification des projets résistants en matière d'éducation. Pour les résistants, juin 1940 marque une césure fondamentale et toute réflexion politique, historique ou culturelle qui négligerait cette donnée est vouée à l'échec. Certes, le poids des traditions et des expériences ne s'est pas subitement effacé. Cependant, le devoir impératif de réflexion et de méditation qui s'impose aux intellectuels résistants a profondément contribué à renouveler, à leurs yeux, les termes des grands débats de l'entre-deux-guerres. Ainsi, il existe au moins un point sur lequel les résistants de l'intérieur s'accordent avec les théoriciens de la Révolution nationale: un événement aussi inouï que la débâcle de 1940 ne peut se résumer à des causes strictement militaires. Il est nécessairement le reflet d'une désagrégation profonde, certains oseront le mot de décadence, de la nation et de ses valeurs fondatrices.

8 En premier lieu, sont dénoncées les innombrables divisions politiques qui ont dissout la cohésion nationale. La prétention à réconcilier les Français explique d'ailleurs les difficultés que certains résistants, surtout en zone Sud, éprouvèrent à rompre définitivement avec Vichy. Non seulement l'ambition de Vichy de réunir les Français a pu, en 1940-1941, séduire certains d'entre eux, mais l'engagement du combat contre Vichy impliquait l'inacceptable perspective d'un nouveau conflit franco-français. Parmi les «divisions stériles » pointées du doigt, les résistants s'accordaient à considérer le conflit religieux qui avait revêtu en France, depuis la seconde moitié du XIXe siècle, la forme d'un conflit scolaire. Le projet unanimiste de la résistance exigeait la recherche d'une solution au conflit scolaire, autrement dit, la réconciliation ou la réunion des « deux jeunesses ».

9 En outre, par un cheminement qui évoque celui des républicains au lendemain de la défaite de 1870, les résistants se sont persuadés que l'école comportait une part de responsabilité dans le désastre de 1940. Mais surgissent alors les limites de la convergence avec le discours pétai-niste : l'école de la République n'est pas coupable au sens où on l'entend à Vichy. Ce qui est en cause, ce n'est pas «l'école sans Dieu », la promotion de "l'esprit de jouissance», le pacifisme des instituteurs ou encore les ravages du syndicalisme. Pour les résistants, si l'école est coupable, c'est de s'être coupée du réel et, surtout, de n'avoir pas suffisamment assuré la formation démocratique des élites.

10 La dénonciation du caractère trop intellectuel et abstrait de l'enseignement de la III République constitue un saisissant lieu commun de la pensée des années de guerre que 
l'on retrouve aussi bien sous la plume des hiérarques de la Révolution nationale que sous celle de la plupart des intellectuels résistants. En outre, l'école de la III ${ }^{\mathrm{e}}$ République, malgré les proclamations et les intentions de ses pères fondateurs, n'était pas suffisamment démocratique. Ce fait est illustré, pour les résistants, par le caractère très élitiste et le recrutement bourgeois de l'enseignement secondaire. Imparfaitement démocratique, l'école de la République a produit des élites puisées parmi des couches trop étroites de la société française, elles mêmes parfois peu convaincues des mérites du système démocratique. Cette élite, qui plus est mal éduquée en raison des vices pédagogiques de l'enseignement français, a fait faillite en 1940 et elle a entraîné le pays dans sa chute. En conséquence, le projet de la résistance vise à démocratiser l'école, à faire en sorte que de son sein émergent les « vraies élites ", c'est-à-dire les élites puisées dans les racines populaires de la nation.

\section{La résistance intérieure et la restauration de la méritocratie républicaine}

11 Sur la question de la réforme du système scolaire, les projets résistants jetaient les éléments d'une réforme radicale, contribuant à dessiner les contours de la grande révolution scolaire des années 1950-1970. Toutefois, la résistance intérieure manifesta davantage de prudence que la France libre. Des propositions le plus souvent disparates, fragmentaires et lacunaires de ses intellectuels (comme Marc Bloch, Claude Bellanger, André Hauriou ou même le communiste Georges Cogniot) émerge davantage la nécessité de réformer la pédagogie plutôt que celle d'engager une vraie démocratisation.

Il est un aspect de l'école de la III $^{\mathrm{e}}$ République qui faisait l'objet d'une critique particulièrement vive de la part des résistants : le «bachotage ». Sous cette expression se cachaient en fait plusieurs réalités : le travail excessif exigé des élèves, la toute-puissance de l'enseignement purement intellectuel, la manie des concours et des classements. Défense de la France, sous la plume du professeur Alphonse Dain, dénonçait la tare de l'enseignement français, insinuant par là même sa responsabilité dans la défaite: «Beaucoup reprochent [à l'Université] d'avoir perdu le pays[...] A l'heure actuelle, la nation manifeste une certaine aversion de l'intellectuel ». La raison de ce rejet résidait dans la " séparation du savoir et de la vie »², elle-même conséquence de l'invasion de l'Université par un scientisme borné et coupé de Dieu. Référence à « l'école sans Dieu » mise à part, le fond de la critique était partagé par d'autres résistants, catholiques ou non. Ainsi, au nom de l'OCM, Claude Bellanger, ancien secrétaire national de la Ligue de l'enseignement, remettait en cause les critères pédagogiques de la $\mathrm{III}^{\mathrm{e}}$ République et accusait un enseignement fondé sur le surmenage et l'accumulation ridicule d'un savoir encyclopédique. Il se prononçait en faveur d'un allégement des programmes et de la définition d'une nouvelle pédagogie qui réduisît le rôle de la mémoire et fit davantage appel à la sensibilité de l'enfant. Il souhaitait également réformer la vie et l'organisation des lycées. Les internats, jugés sinistres, froids et anonymes, devaient disparaître au profit de petits établissements, dirigés par des maîtres pourvus d'un diplôme élevé et capables de créer une ambiance familiale. D'une façon plus générale, les barrières qui existaient entre maîtres et élèves devaient reculer et, comme cela existait dans les collèges britanniques, il était souhaitable que les professeurs vivent avec leurs élèves et participent à la vie sociale de l'établissement. 
13 C'est incontestablement Marc Bloch qui allait le plus loin dans la dénonciation du «bachotage». Le long article que le père de l'école des Annales livra aux Cahiers politiques du CGE, en août 1943, était entièrement bâti sur la dénonciation de l'obsession si française des concours et des examens : «Un mot, un affreux mot, résume une des tares les plus pernicieuses de notre système actuel ; celui du bachotage [...] On n'invite plus les enfants ou les étudiants à acquérir les connaissances dont l'examen permettra, tant bien que mal, d'apprécier la solidité. C'est à se préparer à l'examen qu'on les convie ». Les conséquences de cet état de fait étaient catastrophiques sur le plan physique, mais aussi sur les plans moral et intellectuel, effaçant " toute libre curiosité » au profit "d'une sorte de tremblement perpétuel et de hargne ». Et de conclure irrémédiablement à propos du baccalauréat, archétype français de l'examen : "Certaines condamnations à mort s'imposent. Qui croit encore au baccalauréat, à la valeur de choix, à l'efficacité de cette aléatoire forcerie? "3. Que faire alors? S'inspirant des travaux de Laugier et Piron sur la docimologie, Bloch proposait d'abolir l'échelle française de classement de 0 à 20, qu'il jugeait ridicule, et d'adopter le système anglo-saxon de notation selon cinq grandes catégories échelonnées de "très mauvais » à "très bon ». Cependant, sa terrible philippique négligeait les activités sportives, ignorait les «méthodes actives» et n'avançait que quelques timides réformes: une discipline plus accueillante, des classes moins nombreuses, une gamme d'options plus étendue, des enseignements moins marqués du sceau impitoyable du latin et des mathématiques.

La condamnation du «bachotage » n'était pas sans risque politique, en particulier celui d'être assimilée à la promotion de l'Enseignement Général et Sportif par Vichy. Les rédacteurs d'une Note sur les problèmes de l'enseignement, que l'OCM diffusa en mai 1944, en étaient si conscients qu'ils accompagnaient leur remise en cause du bachotage d'une prudente mise en garde : il ne fallait pas y voir « une critique de la culture à la fois rationaliste et humaniste", mais plutôt un appel à la formation "d'une intelligence créatrice et pas seulement critique $»^{4}$. Cette mise au point presque embarrassée s'expliquait par la date tardive du document. S'il était facile, en 1942, comme le faisait encore Dain, de dénoncer « l'intellectualisme », à partir de 1943, cette dénonciation pouvait prêter à confusion. La politique éducative de Vichy était dorénavant assimilée au STO et réduite à l'action jugée fascisante et "germanique » de Bonnard. L'anti-intellectualisme devenait une arme dangereuse qu'il convenait de manier avec prudence.

Au début de 1944, Essor, le périodique de l'OCM-Jeunes dirigé par Charles Verny et Claude Desjardins, publiait deux articles qui amorçait une Réflexion sur l'école de demain. L'intérêt de ces textes ne résidait pas dans la critique sans surprise du "surmenage ", mais dans la revendication de la participation de l'armée au processus éducatif: "Prenant le jeune Français au sortir de l'école, [l'armée] aura à en faire non seulement un guerrier aguerri, mais un citoyen [...] Elle le rendra fort physiquement, fort moralement, prolongeant ce qu'aura commencé l'école [...] Elle éduquera son esprit à comprendre les idées de base : honneur, discipline, patriotisme $»^{5}$. Les textes d'Essor se démarquaient également par le constat que le partage tacite, élaboré avant la guerre, entre l'école (chargée de l'instruction) et la famille (investie de l'éducation au sens large) avait échoué. De façon encore plus originale, Essor s'en prenait d'ailleurs plus à la famille qu'à l'école et réclamait à l'avenir pour celle-ci une authentique mission éducative. Cette "éducation nouvelle» diffuserait les valeurs traditionnelles de l'école (effort, persévérance, probité, amour du travail), mais aussi celles des groupements de jeunesse (loyauté, émulation cordiale, solidarité), les instituteurs devenant ainsi "maîtres au sens fort du terme» et l'Education nationale se muant en une vaste "école des cadres ${ }^{6}$. Ce plaidoyer en faveur des mouvements de 
jeunesse et de leur méthode, sans surprise sous la plume de jeunes militants dont les liens avec l'ACJF et la JEC étaient forts, permettait d'éloigner le spectre de l'embrigadement par la coopération de l'école et des mouvements de jeunesse. Dans la mesure où la totalité des enfants ne pourraient jamais être encadrés par ces derniers, il était bon que l'école adoptât leurs méthodes afin d'en faire bénéficier le plus grand nombre.

Au total, les conceptions pédagogiques de la résistance permettent de mettre en évidence de fortes convergences. L'intelligentsia française du temps de guerre, de Vichy à Uriage, d'Uriage au maquis, du maquis à Londres, de Londres à Alger, procéda, sous des formes variées et avec des intentions différentes, à une remise en cause commune du primat de l'intellectuel sur le physique, le moral et le manuel.

question de la démocratisation du système éducatif, les intentions des résistants étaient aussi fermes, même si les propositions concrètes qui en découlaient pouvaient paraitre plus timides. Parfois en retrait par rapport aux conceptions des partisans de « l'école unique » des années 1930, les résistants se bornaient à réclamer la restauration du véritable élitisme républicain. Le "Manifeste pour la Nation française», publié par Combaten février 1943, résumait le propos de la résistance, tout en soulignant le glissement vers la définition d'un "socialisme humaniste ": "L'instruction, réellement ouverte à tous, sera inséparable de l'éducation. Elle formera le caractère autant que les intelligences. Elle tirera ainsi du sein de la nation les élites réelles, permettra leur renouvellement constant. Une élite qui ne se renouvelle pas est une élite qui meurt " ${ }^{7}$. En 1944, au moment où s'affirmait l'ambition révolutionnaire de la résistance, l'exigence démocratique fut formulée de façon plus péremptoire. Au printemps 1944, Les Cahiers de Défense de la Francequi présentaient un Projet de constitutionplaçaient le "droit à l'instruction " au rang des droits fondamentaux de l'homme ${ }^{8}$. En mai 1944, la Note sur les problèmes de l'enseignementde l'OCM, plus explicite encore, refusait la perspective d'un enseignement qui perpétuât " les privilèges de classe » et souhaitait, au contraire, voir se "répandre partout l'éducation populaire "'. La Charte du CNR de mars 1944, tout en demeurant dans un flou relatif quant aux modalités d'application, réclamait la démocratisation de l'enseignement, fondée sur «la possibilité effective pour tous les enfants français de bénéficier de l'instruction et d'accéder à la culture la plus développée quelle que soit la situation de fortune de leurs parents, afin que les fonctions les plus hautes soient réellement accessibles à tous ceux qui auront les capacités requises pour les exercer et que soit ainsi promue une élite véritable, non de naissance mais de mérite, et constamment renouvelée par les apports populaires $»^{10}$.

Ce texte, dont la filiation avec le "Manifeste » de Combat est évidente, apparaît comme une sorte de synthèse minimale de la pensée résistante. Eludant délibérément les questions épineuses (école unique, laïcité, avenir des grandes écoles, autonomie de l'Université), il se contentait de réaffirmer les principes très généraux qui avaient fini par rassembler la totalité des résistants.

19 La même prudence se retrouve dans la condamnation des réformes de Vichy. On sait qu'en 1940-1941, le régime de Vichy entreprit une vigoureuse épuration de la fonction publique en général et du monde enseignant en particulier. R. Paxton rappelle que la seule loi antimaçonnique du 13 août 1940 "portant interdiction des associations secrètes" entraina la mise à pied de 1328 instituteurs ${ }^{11}$. Cette épuration, associée à la suppression des écoles normales, apparaissait aux résistants, du moins à la fin de la guerre, comme d'odieuses mesures politiques. A travers l'école de Jules Ferry, c'étaient la République et l'esprit démocratique qui étaient visés. Pourtant, cette défense de l'école primaire n'avait pas été le réflexe premier. Bien plus, jusqu'à la fin de 1942, la plupart des mouvements de 
résistance affichèrent un ton fort critique à l'égard des écoles normales. Cela ne surprend guère dans le cas Défense de la France qui, dans son article de février 1942, déclarait franchement ne pas regretter la disparition des «séminaires laïques » et l'obligation faite par Vichy aux futurs instituteurs d'être munis du baccalauréat : «Soit dit sans méchanceté, le privilège auquel les instituteurs tenaient le plus, celui d'être des primaires, se trouve ainsi gravement atteint $»^{12}$. Cette attitude était plus surprenante sous la plume de Bellanger, ancien instituteur et dirigeant de la Ligue de l'enseignement. Dans les Cahiers de l'OCM, en septembre 1942, il affirmait " [qu]'il n'[était] pas question de rétablir les écoles normales » et proposait de créer des "Instituts pédagogiques " ${ }^{13}$, qui ressemblaient fort à ceux mis en place par Vichy, à ceci près qu'ils seraient rattachés à l'enseignement supérieur et non à l'enseignement primaire.

A partir de 1943, la critique devint beaucoup plus radicale et le rétablissement des écoles normales s'imposa comme une revendication quasi-unanime. Pour autant, le rétablissement ne devait pas s'effectuer à l'identique. Marc Bloch, en août 1943, dans les Cahiers politiques du CGE, dénonçait la suppression des écoles normales et leur remplacement par des instituts pédagogiques. Certes, reconnaissait-il, les écoles normales étaient loin d'être parfaites, mais il fallait préserver et améliorer ce précieux outil pédagogique, plutôt que de le détruire: «Remplacer l'Ecole normale par un passage dans les lycées est un non-sens. Les futurs maîtres y apprennent moins bien ce qu'on leur enseignait mieux dans l'ancienne Ecole ». L'universitaire proposait une solution plus souple qui invitait les instituteurs à " achever leur cycle d'étude par un an de travail très libre dans les universités » ${ }^{14}$. Enfin, l'arrière-pensée politique de Vichy n'échappait à Bloch : la raison principale qui avait conduit Vichy à démanteler les écoles normales n'était pas le souci pédagogique, mais la volonté d'attenter aux principes républicains. Marcel Abraham, l'ancien directeur du cabinet de Jean Zay à l'époque du Front populaire, livrait la même analyse, en mars 1944, dans Les Cahiers de la Libération. Abraham dénonçait le caractère politique des réformes de Vichy, mais il se refusait à absoudre "[ces] séminaire[s] dont l'air confiné pouvait développer ce qu'on appelait l'esprit primaire: une sorte d'assurance satisfaite et de foi sans limite dans un savoir fort limité $»^{15}$. Ainsi la réfutation de Vichy n'impliquait pas, y compris aux yeux d'hommes de gauche, une quelconque restauration intégrale des écoles normales.

\section{La résistance extérieure et l'acceptation de la massification des études}

Le propos de la résistance extérieure était plus audacieux. La vision des Français libres s'inspirait beaucoup plus explicitement des théories de "l'école unique", quitte à les dépasser. Il est difficile de rendre compte de cette différence d'analyse entre les deux résistances. Sans doute faut-il considérer l'influence déterminante au sein des commissions de la France libre, puis du CFLN, d'hommes de gauche, engagés dans ce combat dès avant la guerre. De même, les audacieux projets de démocratisation de l'enseignement conçus, durant la guerre, par les Britanniques et les Américains ont pu inspirer les réformateurs de la France libre, puis du CFLN. La nécessité d'élever le niveau intellectuel de la nation afin de mieux répondre aux défis de la modernisation économique du pays constitue également un thème récurrent chez les gaullistes. Plus profondément, on peut soulever l'hypothèse d'une différence d'appréciation sur le rôle et la responsabilité des élites. Le discours de la résistance intérieure demeure dominé par la problématique de l'élite : une élite a fait faillite en 1940 ; à la résistante, qui se présente 
elle-même comme une élite morale et politique, incombe la mission historique de libérer et de relever le pays, avant d'engager des réformes qui éviteront que ne se reproduise le drame de 1940. Dans cette perspective, le retour au véritable élitisme républicain doit suffire: il ne s'agit pas tant d'élargir les bases de l'élite que de permettre son renouvellement. Les Français libres entendent aller plus loin. Ce qui a fait faillite en 1940, ce ne sont pas seulement l'élite et son mode de formation, c'est la conception même des rapports entre cette élite et la nation. A la vision traditionnelle du filtre joué par les corps intermédiaires, doit se substituer un «lien direct» entre le peuple et ses dirigeants. Ce lien direct exige tout autant le vote des femmes, la réforme des institutions que la refonte complète du système scolaire. Faire en sorte que les élites proviennent des milieux populaires ne suffit pas: il s'agit d'offrir à tous les Français la possibilité effective d'effectuer des études longues.

22 Le projet des Français libres était à la fois simple et révolutionnaire : l'enseignement secondaire devait être unifié et rendu obligatoire. Les réformateurs de la commission Cathala l'exprimaient sans ambages en avril $1942:$ : Par expérience, nous avons la conviction que tous les enfants peuvent suivre des études secondaires ${ }^{16}$. Le Rapport de la commission Durry (août 1944) traçait quant à lui l'ébauche d'une vaste révolution du système éducatif: âge de l'obligation scolaire porté à 16 ou 18 ans; gratuité totale de l'enseignement public; unification des lycées, des écoles primaires supérieures et des cours complémentaires; suppression des barrières propres à l'enseignement secondaire traditionnel ("petites classes» des lycées, examen d'entrée en sixième, examen des bourses, latin obligatoire). Le baccalauréat fit l'objet de débats passionnés. Les plus audacieux proposaient de le supprimer de façon à étendre à l'Université la démocratisation programmée. D'autres réformateurs, plus prudents, entendaient simplement le réformer en introduisant la prise en compte du contrôle continu. Le physicien F. Perrin avança une solution originale qui distinguait sanction des études secondaires (par un diplôme donné) et droit d'entrée à l'Université. Prudemment, la commission laissa cette épineuse question en suspens.

Comparée aux bouleversements qui affectaient l'enseignement secondaire, la réforme du primaire était beaucoup plus modeste. Ainsi, la commission Durry conservait, sans la modifier, la succession des cinq classes primaires. En revanche, la finalité, l'esprit et la pédagogie de cet enseignement étaient appelés à de profondes évolutions. L'idée générale du Rapport Durry était de concevoir désormais le primaire non plus comme un terminus, mais comme l'antichambre du secondaire. En conséquence, les cours complémentaires, les cours supérieurs et les écoles primaires supérieures devaient disparaître. Plus symbolique encore était la décision de supprimer le certificat d'études primaires, ce qui soulignait à nouveau l'audace des réformateurs algérois : Jean Zay, en effet, n'avait pas songé à supprimer le "certif ", mais simplement à le rénover pour en faire un diplôme donnant accès à l'enseignement secondaire. Deux autres vénérables institutions étaient appelées à disparaître : l'examen d'entrée en $6^{\mathrm{e}}$ (la classe de $6^{\mathrm{e}}$ disparaissant elle aussi) et l'examen des bourses qui permettait à quelques élèves méritants du primaire populaire d'accéder au monde du lycée. Dans la mesure où le secondaire devenait obligatoire, ces épreuves n'avaient plus aucune justification.

Restait la délicate question des "petites classes" primaires des lycées. Sous la III République, l'enseignement primaire était divisé entre l'univers populaire des écoles " communales » et le monde culturellement et socialement élitiste des "petits lycées ", classes primaires payantes installées dans les lycées. Malgré leur déclin quantitatif ${ }^{17}$, ces 
classes constituaient une forte entrave à l'établissement de "l'école unique » : en 1939, encore près des deux tiers des lycéens n'avaient pas fréquenté l'école "communale». Même si Jean Zay avait poursuivi l'œuvre d'assimilation de ces classes à l'enseignement primaire engagée par ses prédécesseurs ${ }^{18}$, à la veille de la guerre, la gratuité complète n'était toujours pas établie, la distinction persistait et elle avait même été renforcée par Vichy. Aussi la commission Durry proposait-elle avec fermeté la disparition définitive de ces classes : l'unification du secondaire exigeait l'unification préalable du primaire. Les travaux de la commission inspirèrent directement l'ordonnance du GPRF du 3 mars 1945 qui supprima les " petits lycées».

La commission émettait toutefois le vœu de voir l'esprit de ces classes perdurer et se généraliser à tout l'enseignement primaire. Devenu l'antichambre du secondaire, le primaire ne pouvait conserver son ambition encyclopédique, ce fameux «esprit primaire » qui avait tant été raillé par les beaux esprits de la III République. Le Rapport proposait de recentrer l'enseignement sur les "matières de base» (écriture, calcul, orthographe) et d'alléger ou d'abandonner tout le reste qui serait désormais étudié dans le secondaire. A l'inverse, la commission appelait la multiplication «de nouveaux moyens d'expression ${ }^{19}$ (comme l'éducation physique, la musique, la peinture, les travaux manuels) et surtout l'éclosion d'une nouvelle méthode qui s'inspirait directement des théories de la pédagogie active des années 1930. Plus précisément, le Rapport souhaitait développer «le travail en équipe, les contacts entre l'école et la vie quotidienne». Il désirait "encourager à l'imagination par la composition libre, en un mot, voir les enfants acquérir une autonomie graduelle $»^{20}$. Sur ce point encore, la dette envers le Front populaire était forte : à la rentrée 1936, Jean Zay avait lancé l'expérience modèle d'une demi-journée hebdomadaire d'éducation physique et institué une autre demi-journée consacrée aux « loisirs dirigés » qui devaient développer la culture intellectuelle, esthétique et morale des enfants; en 1938, les programmes de l'enseignement primaire avaient été allégés et l'année scolaire réduite, les vacances d'été commençant le 14 et non plus le 31 juillet. Les instructions aux recteurs d'octobre 1938 évoquaient le refus de la «méthode d'autorité» au profit de la " méthode active » et de "l'esprit libéral "21, La commission Durry proposait de généraliser cette pédagogie, estimant comme Jean Zay en son temps, que réforme politique et réforme pédagogique allaient nécessairement de pair.

Le souci d'éviter toute restauration à l'identique se retrouvait à propos de la formation des maîtres. A l'unisson de la politique du CFLN, la commission prit fermement la défense du corps enseignant contre les attaques de Vichy. Aussi les écoles normales, symbole de l'esprit républicain, furent-elles restaurées sans attendre la Libération par l'ordonnance du CFLN du 4 septembre 1943. Toutefois, les réformateurs algérois, à l'image des résistants de l'intérieur, entendaient rénover autant que restaurer ces séminaires républicains. Ils émettaient le vœu que soient enfin distinguées formation intellectuelle et formation pédagogique et proposaient que les futurs instituteurs suivissent des études secondaires complètes, c'est-à-dire qu'ils fussent munis du baccalauréat. Une fois leur formation intellectuelle achevée dans les lycées, les futurs instituteurs entreraient, sur concours, dans les écoles normales, transformées en "Ecoles pédagogiques", dont les études dureraient deux ans et où leur serait dispensée la formation professionnelle. Une nouvelle fois, les bouleversements étaient considérables. L'instituteur ne débuterait plus à 18 ou 19 ans, mais au minimum à 21 ans, après des études secondaires complètes. De même, les écoles normales devenaient des établissements supérieurs, ce qui constituait une petite révolution culturelle. Jean Zay, en 1937, avait dû reculer devant la fronde du 
SNI quand il avait proposé que les écoles normales fussent dépouillées d'une partie de leur fonction enseignante. Le ministre de Léon Blum avait imaginé, en effet, que les futurs instituteurs suivraient, jusqu'au baccalauréat, les cours des lycées, les écoles normales étant alors réduites au rôle d'internats pour élèves boursiers. Dans un second temps, ces dernières récupéreraient leurs élèves et les formeraient, en deux ans, au métier d'instituteur. La réforme d'Alger, plus habile, évacuait cette période de «latence » des écoles normales. De son côté, Vichy avait, certes, mené à bien cette réforme, mais dans une perspective anti-primaire qui n'était pas celle de Durry et de Capitant.

Fallait-il pour autant offrir une formation identique aux futurs instituteurs et aux futurs professeurs? Au sein de la commission, les représentants de l'enseignement primaire, comme Boissonnet, secrétaire de la Fédération de l'enseignement pour l'Afrique du Nord, défendaient cette solution qui faisait écho à une ancienne revendication du SNI : une fois la formation des maitres des deux degrés harmonisée, il deviendrait difficile de refuser l'assimilation des statuts. La commission repoussa cette perspective au profit de solutions confuses qui masquaient mal son embarras. On pouvait concevoir que les «Ecoles pédagogiques" fussent communes, après le baccalauréat, à tous les candidatsenseignants : au terme des deux années de formation, ceux qui se consacreraient à l'enseignement primaire arrêtaient leurs études, alors que les futurs professeurs gagnaient l'Université où ils passaient une licence, suivie d'un concours de recrutement. Toutefois le Rapport n'excluait pas que les «Ecoles pédagogiques » fussent réservées aux seuls instituteurs et que les futurs professeurs suivissent un cursus strictement universitaire, ponctué de stages pédagogiques dans les « Ecoles pédagogiques ». Ces deux solutions, qui ménageaient les revendications des syndicats enseignants, laissaient subsister de larges zones d'ombre. Ainsi, si l'on suivait la première, était-il raisonnable de demander aux futurs professeurs de lycée de commencer leurs études par la formation pédagogique pour les achever par la formation intellectuelle? Quoiqu'il en soit, et même si elle souhaitait percer « une large brèche dans les vieilles cloisons [...] pour vivifier notre corps enseignant $~^{22}$, la commission maintenait la séparation entre les deux corps enseignants. Plus généralement, de la disparition du certificat d'études et des EPS à la transformation des écoles normales en établissements supérieurs, les réformateurs du CFLN signifiaient le recul de l'enseignement primaire. Pour la plupart professeurs de lycée ou d'Université, ils dessinaient une vaste réforme dont l'épicentre autant que le modèle étaient l'enseignement secondaire. La démocratisation, synonyme d'uniformisation, exigeait le sacrifice de la singularité de l'univers primaire.

\section{Jalons d'une continuité}

Présenté ici à grands traits, le projet éducatif et culturel de la résistance s'inscrit dans une subtile dialectique de continuité et de rupture. Par bien des aspects, les projets résistants semblent prolonger les réflexions de la période du Front populaire. Pourtant, la défaite introduit une césure radicale. Certains projets, que l'on pourrait croire tout droit sortis des cartons de 1936 ont, en fait, changé de signification après la défaite de 1940. Ainsi en va-t-il des motivations de la politique de la jeunesse : à la volonté d'organiser les loisirs se substitue le souci de forger un nouveau sentiment national. La descendance des projets résistants révèle des logiques tout aussi singulières. En matière de réforme de l'enseignement, l'historiographie souligne le rôle de la commission Langevin-Wallon dont le rapport, présenté en 1947, aurait marqué le point de départ du grand ébranlement du 
système scolaire. On sait, pourtant, que les prudents gouvernements de la Troisième Force, peu soucieux de susciter des désaccords entre les partis qui les soutenaient, s'empressèrent de ranger de côté ce document et de renvoyer sine dieson application. Le compagnonnage de route avec le parti communiste de ses deux principaux inspirateurs, au moment où la guerre froide imposait de sévères reclassements politiques, facilita d'ailleurs l'enterrement du projet Langevin-Wallon. Singulièrement, c'est à la V $\mathrm{V}^{\mathrm{e}}$ République gaullienne que revint la tâche de faire entrer dans la réalité les propositions des sympathisants communistes de 1947 (loi Berthoin de 1959, loi Fouchet de 1963). La contradiction n'est qu'apparente et disparaît si l'on accepte de relativiser l'importance historique du rapport Langevin-Wallon. La plupart de ses propositions, présentées en 1947 comme inspirées par le parti communiste (qui d'ailleurs s'en démarqua), étaient, en fait, contenues dans le rapport Durry d'août 1944 et déjà en germe dans les travaux de la très gaullienne commission Cathala de 1942-1943. Le paradoxe est d'autant plus piquant que les propositions communistes en la matière formulées pendant la guerre par Georges $\operatorname{Cogniot}^{23}$ étaient nettement en retrait par rapport aux audacieuses perspectives dessinées par la commission Durry.

L'analyse des projets de la résistance révèle ainsi de fortes continuités historiques. Bien des aspects de la politique des débuts de la $\mathrm{V}^{\mathrm{e}}$ République, de la politique algérienne à la refonte des institutions, de la réforme scolaire à la politique économique et sociale, gagnent en compréhension confrontés aux projets et réalisations de la France libre et du CFLN, véritables « bancs d'essai » du gaullisme.

\section{NOTES}

1. La substance de cette communication est extraite d'un ouvrage à paraître, les enfants de la défaite, aux Presses de Sciences Po, en 1998.

2. «Réflexions sur l'éducation ", in Défense de la France, février 1942, º 19.

3. "Sur la réforme de l'enseignement", in Les Cahiers politiques, août 1943. On peut lire ce texte dans la réédition de L'étrange défaite, Paris, Gallimard-Folio, 1990, p. 254-269.

4. AN, 72 AJ 67.

5. Essor, février 1944.

6. Essor, mars 1944.

7. Combat, février 1943. Le « Manifeste » fut rédigé, à l'été 1942, par Henri Frenay, Claude Bourdet et André Hauriou.

8. Les Cahiers de Défense de la France, printemps 1944. La collection de ce périodique, conservée à la Bibliothèque nationale, n'est pas datée. Le contexte incline toutefois à penser que ce Projet de constitution fut rédigé peu avant le débarquement.

9. $\mathrm{AN}, 72 \mathrm{AJ} 67$.

10. WIEVIORKA O. \& PROCHASSON C., La France du XX siècle, Paris, Le Seuil, 1994, p. 400.

11. PAXTON R.O., La France de Vichy, Paris, Le Seuil, 1973, p. 155.

12. «Réflexions sur l'éducation ", in Défense de la France, février 1942, N 19.

13. "Pour un ministère de la vie culturelle », in Les Cahiers, septembre 1942.

14. «Sur la réforme de l'enseignement ", in Les cahiers politiques, août 1943. 
15. «Vichy contre l'enseignement national », in Les Cahiers de la Libération, mars 1944.

16. AN, $71 \mathrm{AJ} 62$.

17. En 1937, elles n'accueillaient plus que $1,4 \%$ des effectifs du primaire.

18. La «commission de l'école unique» de 1924 avait proposé de transformer les "petites classes » en écoles primaires. En 1926, le statut des enseignants et les programmes des «deux écoles primaires » avaient été unifiés. L'arrêté du 22 mai 1937 avait rattaché les "petits lycées » à la direction de l'Enseignement du premier degré et le budget de 1937 avait engagé le processus de gratuité de ces classes.

19. AN, $71 \mathrm{AJ} 63$.

20. Ibid.

21. Cité par ORY P., La Belle illusion, Paris, Plon, 1994, p. 625.

22. AN, 71 AJ 63.

23. Voir, en particulier l'Esquisse d'une politique française de l'enseignement, rédigée à l'été 1943 par le député communiste de la Seine et diffusée par la presse du PCF et du Front national.

\section{RÉSUMÉS}

Non disponible

Not available

INDEX

Keywords : branch of power, education, french resistance, teaching

Mots-clés : enseignement, France, institution, résistance

\section{AUTEUR}

\section{JEAN-FRANÇOIS MURACCIOLE}

Université Paul Valéry de Montpellier 